\title{
Re: Aminsharifi et al., Major Complications and Adverse Events Related to the Injection of the SpaceOAR Hydrogel System Before Radiotherapy for Prostate Cancer: Review of the Manufacturer and User Facility Device Experience Database (From: Aminsharifi A, Kotamarti S, Silver D, et al., J Endourol 2019;33:868-871; DOI: 10.1089/end.2019.0431)
}

\author{
Richard K. Babayan, MD, ${ }^{1}$ Michael L. Steinberg, MD, ${ }^{2}$ and Larry E. Miller, PhD ${ }^{3}$
}

\section{Dear Editor:}

$\mathbf{W}$ E READ WITH INTEREST the article entitled, "Major Complications and Adverse Events Related to the Injection of the SpaceOAR Hydrogel System Before Radiotherapy for Prostate Cancer: Review of the Manufacturer and User Facility Device Experience Database," by Aminsharifi et al. ${ }^{1}$ The authors queried the MAUDE database and summarized information in medical device reports (MDRs) related to the SpaceOAR perirectal hydrogel spacer, a device intended to reduce rectal toxicity by increasing the space between the prostate and rectum in patients receiving radiation therapy for prostate cancer. They concluded that the number of MDRs associated with this device was increasing over time and that several unique complications were reported within the MDRs that were not identified as potential risks by the manufacturer. In this letter, we present information that challenges the authors' conclusions.

Regarding the increasing number of MDRs since the SpaceOAR device received Food and Drug Administration (FDA) approval in 2015, it is intuitive that the number of MDRs would increase over time concomitant with increasing device use. However, it is misleading to suggest that the complication rate is increasing without accounting for the number of cases performed. We contacted the manufacturer of the SpaceOAR device (Boston Scientific Company) who provided us with the number of SpaceOAR cases that were performed in the United States annually since 2015. We then calculated the rate of SpaceOAR
MDRs adjusted for device usage (Table 1). These results demonstrate that the number of MDRs has been increasing proportionately with device usage and, consequently, the rate of MDRs has remained relatively constant over time, ranging from 0.3 to 0.6 MDRs per 1000 SpaceOAR cases performed.

The authors also state that several unique adverse events reported within MDRs were not acknowledged as potential risks on the manufacturer's website. According to the Instruction's for Use, Potential complications that may be associated with the use of SpaceOAR System include, but are not limited to: pain associated with SpaceOAR hydrogel injection; pain or discomfort associated with SpaceOAR

Table 1. Medical Device Reports with SpaceOAR Perirectal Hydrogel Spacer

Since Commercialization in the United States

\begin{tabular}{cccc}
\hline Year & $\begin{array}{c}\text { No. of } \\
\text { MDRs }\end{array}$ & $\begin{array}{c}\text { No. of Space } \\
\text { OAR cases }\end{array}$ & $\begin{array}{c}\text { MDRs per 1000 } \\
\text { SpaceOAR cases }\end{array}$ \\
\hline 2015 & 1 & 1802 & 0.6 \\
2016 & 2 & 5544 & 0.4 \\
2017 & 3 & 9890 & 0.3 \\
2018 & $14^{\mathrm{a}}$ & 22,225 & 0.6 \\
$2019^{\mathrm{b}}$ & 5 & 8361 & 0.6 \\
\hline
\end{tabular}

${ }^{\mathrm{a}}$ Indicates 11 MDRs involving 14 cases.

${ }^{\mathrm{b}}$ Reported through March 31, 2019.

$\mathrm{MDR}=$ medical device report.

\footnotetext{
${ }^{1}$ Department of Urology, Boston University School of Medicine/Boston Medical Center, Boston, Massachusetts.

${ }_{3}^{2}$ Department of Radiation Oncology, David Geffen School of Medicine at UCLA, Los Angeles, California.

${ }^{3}$ Miller Scientific, Johnson City, Tennessee.
}

(C) Richard K. Babayan et al. 2020; Published by Mary Ann Liebert, Inc. This Open Access article is distributed under the terms of the Creative Commons Attribution Noncommercial License (http://creativecommons.org/licenses/by-nc/4.0/) which permits any noncommercial use, distribution, and reproduction in any medium, provided the original author(s) and the source are cited. 
hydrogel; needle penetration of the bladder, prostate, rectal wall, rectum, or urethra; injection of SpaceOAR hydrogel into bladder, prostate, rectal wall, rectum, or urethra; local inflammatory reactions; infection; injection of air, fluid, or SpaceOAR hydrogel intravascularly; urinary retention; rectal mucosal damage, ulcers, necrosis; bleeding; constipation; and rectal urgency. ${ }^{2}$ Regarding MDRs, the FDA states, Although MDRs are a valuable source of information, this passive surveillance system has limitations, including the potential submission of incomplete, inaccurate, untimely, unverified, or biased data. In addition, the incidence or prevalence of an event cannot be determined from this reporting system alone due to under-reporting of events, inaccuracies in reports, lack of verification that the device caused the reported event, and lack of information about frequency of device use. ${ }^{3}$ Given that the device labeling appears complete with regard to potential risks with the SpaceOAR device and that information within MDRs cannot reliably establish causality of events, it is unclear what specific risks the authors may suggest adding to the current product labeling.

Identification of potential safety risks attributable to medical devices is an important endeavor and, for this, we applaud the authors for presenting this work. We agree that medical device manufacturers should be vigilant in revising product labeling in collaboration with the FDA should new important risks to patient safety be identified. However, we feel that the authors' implications of increasing adverse event rates and inadequate risk labeling with the SpaceOAR device are unjustified.

\section{Author Disclosure Statement}

R.K.B. served on the Clinical Events Committee (CEC) for the SpaceOAR randomized pivotal trial (ClinicalTrials.gov Identifier: NCT01538628). Michael Steinberg discloses consultancy with Boston Scientific and Vision RT and received honorarium from ViewRay. L.E.M. discloses consultancy with Boston Scientific.

\section{References}

1. Aminsharifi A, Kotamarti S, Silver D, et al. Major complications and adverse events related to the injection of the SpaceOAR hydrogel system before radiotherapy for prostate cancer: Review of the Manufacturer and User Facility Device Experience Database. J Endourol 2019;33:868-871.

2. Augmenix Inc. SpaceOAR System Instruction's for Use. https://2ygv91350b7a2gm5pl1ftyu1-wpengine.netdna-ssl.com/ assets/LCN-80-1010-001-en-Rev-L_SpaceOAR-System-10mLIFU.pdf Accessed October 4, 2019.

3. Food and Drug Administration. MAUDE-Manufacturer and User Facility Device Experience. www.accessdata.fda .gov/scripts/cdrh/cfdocs/cfMAUDE/search.CFM Accessed October 4, 2019.

Address correspondence to:

Larry E. Miller, PhD Miller Scientific

3101 Browns Mill Road, Ste. 6 \#311 Johnson City, TN 37604

E-mail: larry@millerscientific.com 